\title{
Genetic Diversity of Pto-Like Serine/Threonine Kinase Disease Resistance Genes in Cultivated and Wild Strawberries
}

\author{
M. G. Martínez Zamora • A. P. Castagnaro • \\ J. C. Díaz Ricci
}

Received: 30 April 2008/Accepted: 9 June 2008

(C) Springer Science+Business Media, LLC 2008

\begin{abstract}
Degenerate oligonucleotide primers, designed based on conserved regions of several serine-threonine kinases (STK) previously cloned in tomato and Arabidopsis, were used to isolate STK candidates in wild and cultivated strawberries. Seven distinct classes of STKs were identified from three related wild species, i.e., Fragaria vesca, Fragaria chiloensis, and Potentilla tucumanensis, and seven different Fragaria $\times$ ananassa cultivars. Alignment of the deduced amino acid sequences and the Pto R protein from tomato revealed the presence of characteristic subdomains and conservation of the plant STK consensus and other residues that are crucial for Pto function. Based on identity scores and clustering in phylogenetic trees, five groups were recognized as Pto-like kinases. Strawberry Pto-like clones presented sequences that were clearly identified as the activation segments contained in the Pto, and some of them showed residues previously identified as being required for binding to AvrPto. Some of the non-Pto-like kinases presented a high degree of identity and grouped together with B-lectin receptor kinases that are also involved in disease resistance. Statistical studies carried out to evaluate departure from the neutral theory and nonsynonymous/synonymous substitutions suggest that the evolution of STK-encoding
\end{abstract}

M. G. Martínez Zamora · J. C. Díaz Ricci $(\bowtie)$

INSIBIO (CONICET-UNT), Departamento de Bioquímica de la Nutrición e Instituto de Qca Biológica "Dr. Bernabé Bloj"

(UNT), Chacabuco 461, 4000 Tucuman, Argentina

e-mail: juan@fbqf.unt.edu.ar

\footnotetext{
A. P. Castagnaro

Sección Biotecnología, Estación Experimental Agroindustrial O. Colombres-Unidad Asociada al INSIBIO, CC No. 9, 4101 Las

Talitas, Tucuman, Argentina

e-mail: atilioc@fbqf.unt.edu.ar
}

sequences in strawberries is subjected mainly to a purifying selection process. These results represent the first report of Pto-like STKs in strawberry.

Keywords Disease resistance genes .

Serine-threonine kinase · Pto · Fragaria spp.

B-lectin receptor kinases · Genetic diversity

\section{Introduction}

The strawberry is an important horticultural crop in many tropical, subtropical, and temperate areas, and its production has increased from $0.75 \mathrm{mt}$ (million tons) in 1961 to more than $3.5 \mathrm{mt}$ in 2004 (FAO of the United Nations). Strawberry crop values, based on gross return per hectare, are among the highest of agricultural commodities. Nevertheless, yields are strongly threatened by fungal diseases and pests, forcing the intensive use of chemical products to control them. This scenario places the sustainability and environmental impact of strawberry production under serious concern by health and environmental agencies due to the use of pesticides containing volatile organic compounds such as methyl bromide (Morrisette 1989). The main strategy used by plants to defend themselves against attack of pathogens is the hypersensitive response (HR). This reaction is characterized by localized and rapid cell death around the site of infection (Martin et al. 2003), which is usually followed by a further secondary resistance reaction, termed systemic acquired resistance (SAR), induced in distal noninfected tissues (Ryals et al. 1996). The basis of plant disease resistance involving HR was early explained by Flor (1956), who proposed a gene-for-gene model that implies the interaction of a specific pathogen avirulent gene product (Avr) with a plant dominant resistance gene product (R). 
Resistance gene-mediated breeding is the most suitable strategy to control diseases. Sources of resistance to the most devastating crop pathogens (i.e., genera Colletotrichum and Botrytis) are well documented (Smith and Black 1990; Denoyes-Rothan et al. 2005), and molecular markers of disease resistance in strawberry have been reported (Haymes et al. 1997; Lerceteau-Kohler et al. 2002). However, resistance breeding in commercial strawberries is difficult because of the octoploid genetic structure. Alternatively Agrobacterium-mediated genetic transformations have been developed, with the aim of incorporateing resistance traits (Vellicce et al. 2006; Qin et al. 2008), but no strawberry $R$ gene has been isolated so far.

Recently, an increasing number of plant disease resistance $(R)$ genes from different species have been identified by transposon tagging or map-based cloning. Sequence comparisons among these genes revealed a remarkable conservation of structural features despite the diversity of the pathogens they interact with (Martin 1999; Ellis et al. 2000). Hammond-Kosack and Jones (1997) have classified $R$ genes into eight classes based on the presence of conserved domains, namely, leucine-rich repeats (LRRs), nucleotide binding sites (NBSs), leucine zippers (LZs), and protein kinases (PKs). The most prevalent class of cloned genes is the NBS-LRR class (McHale et al. 2006), which can be categorized into the TIR and non-TIR subclasses, based on the presence of an N-terminal domain with homology to the receptors of the animal innate immunity factors Toll and interleukin-1 (Parker et al. 1997) and the presence of an LZ motif, respectively (Pan et al. 2000; Dangl and Jones 2001).

The tomato Pto gene belongs to another $R$ gene family characterized as presenting a serine-threonine kinase (STK) domain. Pto is the first plant $R$ gene cloned that functions in a gene-for-gene manner (Martin et al. 1993) and encodes a cytoplasmic STK that interacts with the avirulence proteins avrPto and avrPtoB from Pseudomonas syringae pathovar tomato conferring HR-mediated resistance (Kim et al. 2002). Transgenic tomato plants overexpressing Pto under a strong promoter (35S) displayed AvrPto-independent resistance not only to Pseudomonas strains but also to many other bacterial and fungal pathogens (Tang et al. 1999), making the Pto gene an important candidate for broad-spectrum resistance molecular breeding. Although proteins with STK domains may also be involved in other cellular processes such as cell growth and development (Rudrabhatla et al. 2006), the STK domain of Pto-like proteins evolved differently and groups separately in phylogenetic trees (Vallad et al. 2001).

The LRR sequences mentioned above are known to be involved in protein-protein interactions and are the logical candidates for mediating the recognition of Avr molecules (Fluhr 2001). In this regard the Pto gene is unique among the cloned $R$ genes, for it lacks the LRR motif, although it interacts directly with AvrPto as reported by Scofield et al. (1996). Nevertheless, Pto-mediated resistance requires an LRR protein that is encoded by the Prf gene located within the Pto locus. Perhaps Pto and AvrPto form a complex that is able to interact with Prf (Scofield et al. 1996; Kim et al. 2002).

Analysis of the Pto sequence revealed nine major conserved subdomains (I-IX) that are important for catalytic activity. The activation domain, between amino acid 182 and amino acid 211, contains residues that play a key role in recognition and interaction with other proteins (Sessa et al. 2000a, b). The most important residues are T204 and Y207 (inside the $\mathrm{P}+1$ loop), because they are required for the specific recognition and binding of Pto-AvrPto (Frederick et al. 1998). The activation domain also contains four autophosphorylation sites that appear to be required for kinase activity or physical interaction with proteins. Among these, the residue S198 is required for elicitation of the HR (Sessa et al. 2000b).

The evolution of plant genomes is characterized by the occurrence of multiple gene duplication events that may occur through unequal recombination. Like other plant genes, $R$ genes are members of multigene families, suggesting that gene duplication and subsequent diversification are usual mechanisms in plant $R$ gene evolution (Richter and Roland 2002). The Pto gene belongs to a small gene family, consisting of six members spanning a $60-\mathrm{kb}$ region on tomato chromosome 5 (Martin et al. 1993). Pto paralogues share 78-91\% nucleotide identity with Pto, and although most of these paralogues are functional PKs, none of them encode recognition of avrPto and avrPtoB (Chang et al. 2002; Kim et al. 2002). It is presumed that duplication and diversification of the Pto gene family have led to the generation of alternative recognition specificities. One member of this family, the Fen gene, encodes for an STK that is $87 \%$ identical to Pto and participates in the same signal transduction pathway leading to HR, but is activated by a different signal (Martin et al. 1994; Loh and Martin 1995).

Many studies have been carried out to test the hypothesis of host-parasite coevolution and functional adaptation of the Pto gene. Rose et al. (2007) studied sequence variation among Pto alleles of seven species of wild tomato (Lycopersicon) in comparison to the variation observed at 14 non-R-gene loci. Among the Pto alleles, they found higher levels of nonsynonymous and similar levels of synonymous polymorphisms compared to the non- $R$-gene loci, suggesting that these changes may be mediated by pathogen selection. However, they detected a lower frequency of amino acid substitutions that negatively affect Pto function compared to silent polymorphisms. They also observed that a larger proportion of the Pto protein can tolerate more amino acid variation than the non- $R$ genes and concluded that it is possible that the diversity observed is neutral with respect to Pto function. The latter further 
suggests that the evolution of the Pto locus may be influenced by a mixture of both purifying and balancing selection (Rose et al. 2007).

Bernal et al. (2005), by means of DNA-shuffling experiments of Pto and four of its paralogues, generated chimeric Pto proteins and studied their ability to recognize AvrPto and AvrPtoB. In those experiments they identified amino acid positions such as Ser76 and Gly78 that were critical for the recognition specificity. Since particular amino acid substitutions in Pto can change the recognition pattern, enabling differentiation of these two avirulence proteins, it is possible that the amino acid variation among Pto alleles could be maintained by quantitative or qualitative specificity differences or binding ability to AvrPto, AvrPtoB, or other avirulence proteins. This "multiple recognition" ability may be a consequence of the host's adapting to recognize different pathogen effector molecules. This evolutionary scenario could be important in the case of this class of $R$ genes that directly interact with the Avr gene product (Bernal et al. 2005).

Recently, use of the polymerase chain reaction (PCR) with degenerate primers targeted at short conserved domains proved to be an efficient method to identify $R$ gene homologues. This method has been used succesfully in potato (Leister et al. 1996), soybean (Yu et al. 1996; Kanazin et al. 1996), lettuce (Shen et al. 1998), Arabidopsis thaliana (Speulman et al. 1998; Aarts et al. 1998), and Brassica spp. (Joveux et al. 1999), among many others, to isolate the NBS-LRR type of $R$ genes. Also, using the same approach, Pto-like sequences have been isolated from Solanum (Vleeshouwers et al. 2001), bean (Vallad et al. 2001), and banana (Peraza-Echeverría et al. 2007).

In our laboratory, using degenerate primers designed by Leister et al. (1996), we have isolated and characterized NBS-LRR analogues from wild and cultivated strawberries. However, this experimental approach failed to detect any representative of this gene family in some cultivars of Fragaria $\times$ ananassa and some of the wild relatives present in our bank (Martínez Zamora et al. 2004).

The aim of this work was, therefore, to move one step forward in the investigation of strawberry $R$ genes by cloning STK sequences from wild and cultivated strawberries, to identify putative Pto orthologues and study their molecular features, evolution, and phylogenetic relationships.

\section{Materials and Methods}

\section{Plant Material and DNA Extraction}

DNA from Fragaria $\times$ ananassa cultivars Camarosa, Gaviota, Oso Grande, Sweet Charlie, Pájaro, Milsei Tudla, breeding line US159 (Galletta et al. 1993), and specific accessions of the related wild species Fragaria vesca, Fragaria chiloensis, and Potentilla tucumanensis were used for Pto-RGA isolation. Genomic DNA was extracted from young leaflets using the GenElute Plant Genomic DNA Miniprep Kit (Sigma).

\section{Degenerate PCR}

Degenerate PCR primers used were those designed by Vallad et al. (2001) based on conserved regions of amino acid identity between the STKs Pto, Fen, and Ptil from tomato and MHK and APK1 from Arabidopsis thaliana. Primer sequences were P3 (subdomain I), $5^{\prime}$ - TNGGNSANGGNGKNTTYGG-3' ${ }^{\prime}$, and P2R (subdomain IX), 5' - ACNCCRAANGARTANACRTC$3^{\prime}$. PCRs were carried out in a total volume of $50 \mu$ l containing $200 \mathrm{ng}$ of template DNA, $50 \mathrm{mM} \mathrm{KCl}, 10 \mathrm{mM}$ Tris- $\mathrm{HCl}(\mathrm{pH}$ 8.4), $2 \mathrm{mM} \mathrm{MgCl}$, a $5 \mu \mathrm{M}$ concentration of each primer, a $0.3 \mathrm{mM}$ concentration of each dNTP, and $2 \mathrm{U}$ of Taq DNA polymerase (Promega, Madison, WI, USA). PCR was performed in a PTC-100 thermal cycler (MJ Resarch, Inc.). Cycling conditions included an initial denaturation step of $3 \mathrm{~min}$ at $95^{\circ} \mathrm{C}$, followed by 35 cycles of $45 \mathrm{~s}$ of denaturation at $95^{\circ} \mathrm{C}, 45 \mathrm{~s}$ of annealing at $45^{\circ} \mathrm{C}, 1 \mathrm{~min}$ of elongation at $72^{\circ} \mathrm{C}$, and a final extension step of $18 \mathrm{~min}$ at $72^{\circ} \mathrm{C}$. Different concentrations of genomic DNA and primers were tested to optimize the reaction.

\section{Cloning and Sequencing of PCR Products}

Amplification products were separated by electrophoresis on $1.5 \%$ TBE-agarose gels and stained with ethidium bromide for visualization. Bands were excised from the gel and purified using the GFX PCR DNA and Gel Band Purification Kit (Amersham Biosciences, UK). Each purified band, presumably containing different products of similar sizes, was cloned using the pCR2.1 plasmid of the TopoTA Cloning Kit (Invitrogen) following the methods recommended by the manufacturer and transformed into Escherichia coli DH5- $\alpha$ competent cells. Ten recombinant bacterial colonies obtained from each ligation event were isolated. Recombinant plasmid was extracted using the Wizard plus SV Minipreps DNA Purification System (Promega) and digested with $E c o$ RI to verify the presence of the expected insert. Sequences of PCR products were determined using an ABI Prism 377 DNA sequencer (Applied Biosystems, Foster City, CA, USA). The nucleotide sequences of strawberry STKs have been deposited in the GenBank Database under accession numbers EU272664-EU272674.

Sequence Edition, Similarity Searches, and Alignments

Clones were trimmed of vector sequence contamination using VecScreen at NCBI, and the ORF finder program 
was used to search ORFs of strawberry STK (http:// www.ncbi.nlm.nih.gov/). Assembly of DNA sequences and translation to the predicted amino acid sequence were performed using the DNAMAN software (version 4.03), and intron search (putative splicing sites) was performed with the NETPLANTGENE program (Hebsgaard et al. 1996). Identity of STKs from strawberry was confirmed by comparisons of DNA and amino acid sequences with the GenBank NR database using BLASTX and BLASTP (Altschul et al. 1990) algorithms. Determination of conserved structural motifs in STK sequences was carried out with PROSITE (Hofmann et al. 1999).

\section{Evolutionary Analysis}

Multiple sequence alignments were performed with Clustal X (Thompson et al. 1997) and edited with the BOXSHADE server (version 3.21; http://www.ch.embnet.org/software/ BOX_form.html). A codon-based DNA alignment was constructed and manually checked for molecular evolutionary analysis. Rates of synonymous versus nonsynonymous substitution were calculated using the Nei-Gojobori method (1986) with the SNAP program via http://www.hiv-web. lanl.gov/SNAP/WEBSNAP/SNAP.html (Korber 2000).

GeneConv software (http://www.math.wustl.edu/ saw yer/geneconv) was used to identify apparent gene conversion events (Sawyer 1989). GeneConv compares an alignment of at least three nucleotide sequences, identifies sites of paralogous variation among them, and, for each pair, measures the length of stretches of shared variants. The length of these stretches is compared to a simulated distribution (10,000 iterations) of the same number of sequences with a similar average density of variation and used to calculate a Bonferroni-corrected $p$-value. A low $p$ value indicates a high probability that gene conversion occurred. Two neutrality tests (Tajima's [1989] $D$, Fu and Li's [1993] D) were applied with the DNASP computer program version 3.14 (Rozas et al. 2003) to find evidence of positive selection in our candidate gene set.

The amino acid alignments were tested for phylogenetic information by the least biased maximum likelihood (ML) approach with the TREE-PUZZLE software package (Schmidt et al. 2002) using the Dayhoff amino acid substitution model (Dayhoff et al. 1978). This package was used because it allowed us to incorporate the among-site rate variation and gamma-distributed rates of heterogeneity among sites into the model. Transition/transversion parameters and amino acid frequencies were inferred from the data set. Branches were calculated without the molecular-clock assumption. Quartet puzzling with 10,000 puzzling steps was applied to choose from the possible tree topologies and to simultaneously infer support values for internal branches.

\section{Results}

Isolation of Pto-Like Resistance Gene Analogues from Strawberry

Amplification of cultivated and wild strawberry genomic DNA with the degenerate primers designed by Vallad et al. (2001) generated main fragments of about $550 \mathrm{bp}$ and fainter bands of a size between 900 and 950 bp. Although the first corresponded to the expected size, we decided to clone both fragments. We observed similar banding patterns in all Fragaria $\times$ ananassa cultivars and all wild strawberry accesions. Cloning and characterization of these amplicons revealed that each band included many STK sequences. Of the 75 putative strawberry STK clones sequenced, 67 had significant BLAST hits in the GenBank NR Database to STK genes and Pto-like RGAs from other species such as Rosa spp., Vitis spp., and Arabidopsis showing high levels of identity (Table 1). One clone presented a sequence coding a putative ORF with homology to many members of the Regulator of Chromosome Condensation (RCC1) family with up to $81 \%$ identity. Thirty-six of these 67 STKs had potential stop codons and/or frame shift mutations, whereas the other 31 presented uninterrupted ORF. No putative splicing sites were detected in these clones.

\section{Diversity Analysis of Strawberry STKs}

A diversity analysis of strawberry STKs was performed with the 31 nucleotide sequences selected. Nucleotide sequence identity among strawberry STKs ranged between $23 \%$ and $100 \%$, while amino acid identity ranged from $39 \%$ to $100 \%$. Compared to the tomato Pto protein, amino acid identity ranged from $27 \%$ to $46 \%$. Additionally, strawberry sequences showed the highest BLAST scores compared with the STKs identified in other plant species, as reported in Table 1. Strawberry STK amino acid sequences were subjected to phylogenetic analysis using the ML approach, along with the corresponding region of the tomato Pto protein and four of its paralogues (Fig. 1).

The tree shows that most strawberry STKs, including representatives of the cultivated and wild species (except Fragaria vesca), grouped in five Pto-like classes (i.e., AE), and the Pto and paralogues grouped together in a separate class constituting a Pto clade. Class F was composed by members of Potentilla tucumanensis and Fragaria vesca, and class $\mathrm{G}$ was composed of clones $>900$ bp long only, and was used to root the phylogenetic tree. Classes B and $\mathrm{C}$ included the majority of Pto-like sequences, but since all clones presented $>97 \%$ identity, they were considered redundant, and only one clone was chosen to represent these classes. Based on the latter criterion, 11 
Table 1 Similarity among serine/threonine kinases (STKs) from strawberry and other species

\begin{tabular}{|c|c|c|c|c|}
\hline Strawberry STK & Highest BLAST P-score & Identity (\%) & Similarity $(\%)$ & e-value \\
\hline ptoOSO-7 & ABV30718; kinase-like protein & 88 & 93 & $2 \mathrm{e}-90$ \\
\hline (A) & Prunus serrrulata & & & \\
\hline ptoPAJ-1 & CAO44912; hypothetical protein & 85 & 93 & $6 e-93$ \\
\hline (B) & Vitis viniferm & & & \\
\hline ptoCAM-7 & AT28297; Pto-like PK & 95 & 98 & $2 e-96$ \\
\hline (C) & Rasa roxburghii & & & \\
\hline PtoCHILO-4 & CAN77769; hypothetical protein & 92 & 95 & $2 e-93$ \\
\hline (D) & Vitis vinifera & & & \\
\hline ptoPOT-6 & ABV30731; kinase-like protein & 89 & 94 & $1 e-85$ \\
\hline (E) & Prunus avium & & & \\
\hline ptoPOT-16 & CAN70210; lectin PK (putative) & 78 & 91 & $9 e-76$ \\
\hline (F) & Vitis vinifera & & & \\
\hline PtoVES-8 & BAE98939; receptor-like PK & 73 & 85 & $5 e-124$ \\
\hline (G) & Arabidopsis thaliana & & & \\
\hline
\end{tabular}

Note: PK, protein kinase. Capital letters in parentheses $(\mathrm{A}-\mathrm{G})$ correspond to different strawberry STK classes

individual clones were selected for codon-based DNA alignment and further evolutionary analysis.

The average nonsynonymous-to-synonymous substitution ratio $\left(\mathrm{K}_{\mathrm{a}} / \mathrm{K}_{\mathrm{s}}\right)$ among triplets encoding the same amino acids determined by SNAP was 0.17. Using GENECONV no statistically significant regions of gene conversion were identified (Sawyer 1989), suggesting that recombination is unlikely to occur within these DNA fragments. Accordingly, we applied the Tajima and the $\mathrm{Fu}$ and $\mathrm{Li}$ tests of selective neutrality, both based on the infinite-site model without recombination and appropriate for short DNA sequences. As indicated by $D$ statistics and their $p$-values $\left(D_{\mathrm{T}}=-0.52, p>0.294\right.$, and $\left.D_{\mathrm{F}}=0.68, p>0.213\right)$, no significant departure from neutral expectations was observed. Taken together, these results indicate that, at the molecular level, natural selection may be acting to remove deleterious mutations (e.g., purifying selection).

\section{Multiple Amino Acid Sequence Alignment and Phylogenetic Analysis}

The region between subdomain I and subdomain IX was used to generate a Clustal X multiple-amino acid sequence alignment between the tomato Pto, Fen, and Ptil proteins and the deduced amino acid sequence of strawberry STKs (Fig. 2). The alignment revealed the conservation of all subdomains expected for a STK fragment isolated with primers $\mathrm{P} 3$ and $\mathrm{P} 2 \mathrm{R}$ and identified specific residues. The plant STK consensus (Hanks and Quinn 1990) was highly conserved with the exception of G228 in ptoCAM-7, P210 in both representatives of class F (ptoPOT-16 and ptoVES3 ), and D182 and G184 in ptoPot-12 from class G. Of the four autophosphorylation sites inside the activation domain
(Sessa et al. 2000b), S198, which is required for elicitation of HR, T195, and T199 are fully conserved in strawberry STKs except for classes F-G, therefore they were considered non-Pto-like STKs. The Pto (T190) autophosphorylation site was replaced by proline in some of the strawberry STKs (Fig. 2).

The evolutionary relationships of strawberry STKs were investigated using phylogenetic reconstructions using the ML approach. To generate a plant a PK phylogenetic tree we performed a Clustal $\mathrm{X}$ alignment with the 11 STKs sequences from strawberry, Pto, Fen, and Pti1 from tomato, 36 STKs sequences from $A$. thaliana characterized by Hardie (1999), and sequences from various plant species that had the best Blast hits against strawberry STKs. The phylogenetic tree constructed based on this alignment confirmed that the seven strawberry sequences from classes A-E clustered with tomato Pto $\mathrm{R}$ protein, its paralogue Fen, and Pto-like sequences from both monocot and dicot species, supporting the designation of these sequences as Pto-like STKs (Fig. 3). The tomato Pti1 kinase is an STK that interacts with Pto but is not a Pto paralogue (Zhou et al. 1997) and is located outside the main group, as expected.

Regarding the non-Pto-like STK sequences included in class $\mathrm{F}$, results show that they display a high identity to putative lectin receptor-like kinases (LecRLKs) from many species and cluster with a characterized LecRLK from Populus nigra, the PnLPK protein (Nishiguchi et al. 2002). Recently, rice LecRLK gene $P i-d 2$, which confers resistance to Magnaporthe grisasea, was cloned and characterized. The latter encodes a receptor-like kinase protein with a predicted extracellular domain of a bulb-type mannose specific binding lectin (B-lectin) and an 


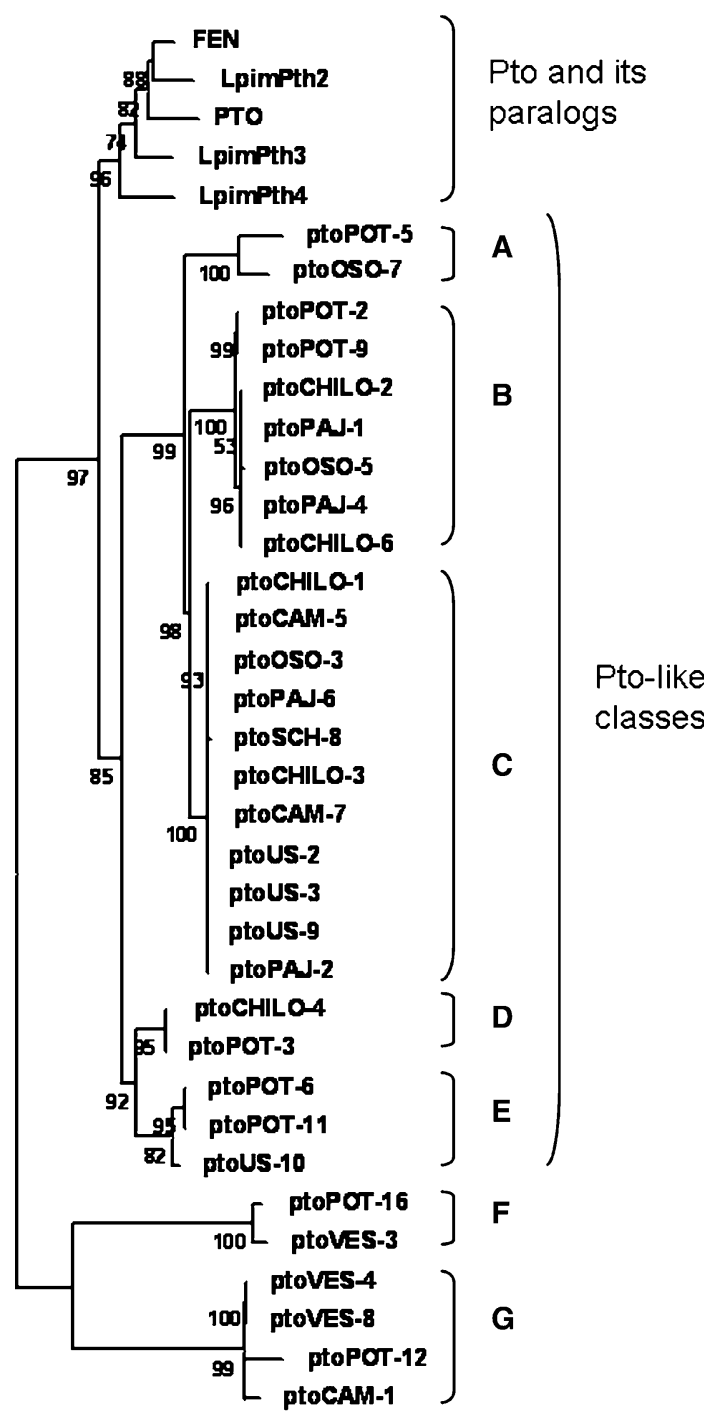

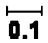

Fig. 1 ML phylogenetic tree computed with TREE-PUZZLE, based on the alignment of the deduced amino acid sequences of strawberry STKs, the tomato Pto $\mathrm{R}$ protein, and its paralogues (GenBank accesion number AF220602). Branches corresponding to strawberry STK classes are labeled A to G. Fragaria $\times$ ananassa cultivars: CAM, Camarosa; PAJ, Pajaro; OSO, Oso Grande; US, line US159. Accessions of the wild species: VES, F. vesca; CHILO, F. chiloensis; POT, Potentilla tucumanensis. The tree was rooted with class G, composed of clones $>900 \mathrm{bp}$ long. Values on tree branches represent the PUZZLE support values, estimated by quartet puzzling (QP) tree search and expressing the QP reliability as a percentage

intracellular STK domain, and it was classified as a novel class of the plant $R$ genes (Chen et al. 2006). Finally, sequences of the 920-bp clones (included in class G) were highly identical and grouped together with the STK domains of many S-receptor like PKs which are known to be involved in the sporophytic self-incompatibility response in Brassica, one of probably many molecular mechanisms by which hermaphrodite flowering plants avoid self-fertilization (Cui et al. 2000).

The sequence characteristics and amino acid residues that proved to be involved in Pto-AvrPto recognition or binding were also compared. The Pto activation domain, located between subdomain VII and subdomain VIII, was present in strawberry Pto-like STKs, and its activation segment (the $\mathrm{P}+1$ loop) is highly conserved (Fig. 2). Further analysis of the Pto-like sequences (classes A-E shown in Fig. 1) is summarized in Table 2. Three of the key residues, K202, Y207, and D209 (in the P + 1 loop), that influence binding properties between Pto and AvrPto are conserved in all strawberry sequences (Frederick et al. 1998). The Pto T204 residue, involved in recognition specificity, is replaced by a serine in all other STKs (Table 2). Residues V55 and H94, important in the interaction with AvrPto, are fully conserved in strawberry Ptolike sequences (Scofield et al. 1996). On the other hand, residues S76 and G78, involved in Avr protein binding specificity (Bernal et al. 2005), present different degrees of conservation, whereas G78 is fully conserved, and S76 presents a degree of variation similar to that observed in Pto and its paralogues. Finally, all strawberry sequences show an insertion of three amino acids at position 159 of the Pto protein (Table 2).

\section{Discussion}

Using the combination of degenerate primers $\mathrm{P} 3$ and $\mathrm{P} 2 \mathrm{R}$, designed to anneal the sequence of subdomains I and IX of the serine/threonine domain of tomato and Arabidopsis, we were able to isolate and clone seven unique Pto-like sequences and four other STK sequences from wild and cultivated strawberries. The number of unique Pto-like sequences obtained is lower than that of NBS-LRR sequences previously isolated in our lab (Martínez Zamora et al. 2004). Nevertheless, we may hypothesize that the Pto-like clones isolated in this study represent a significant proportion of the total number of these sequences in the strawberry genome, since there is evidence that the 15 Ptolike sequences identified in the Arabidopsis genome represent approximately $10 \%$ of the NBS-LRR type of resistance proteins (http://www.niblrrs.ucdavis.edu).

All predicted products of cloned strawberry sequences of the STK class contain nine subdomains (I-IX) and the plant STK consensuses are fully conserved in the Pto-like families, suggesting that they are likely to encode active kinases. Additionally, key residues of the corresponding activation domain in tomato Pto are highly conserved, especially Y207, present in all strawberry STKs. Pedley and Martin (2003) reported that mutation of this residue 


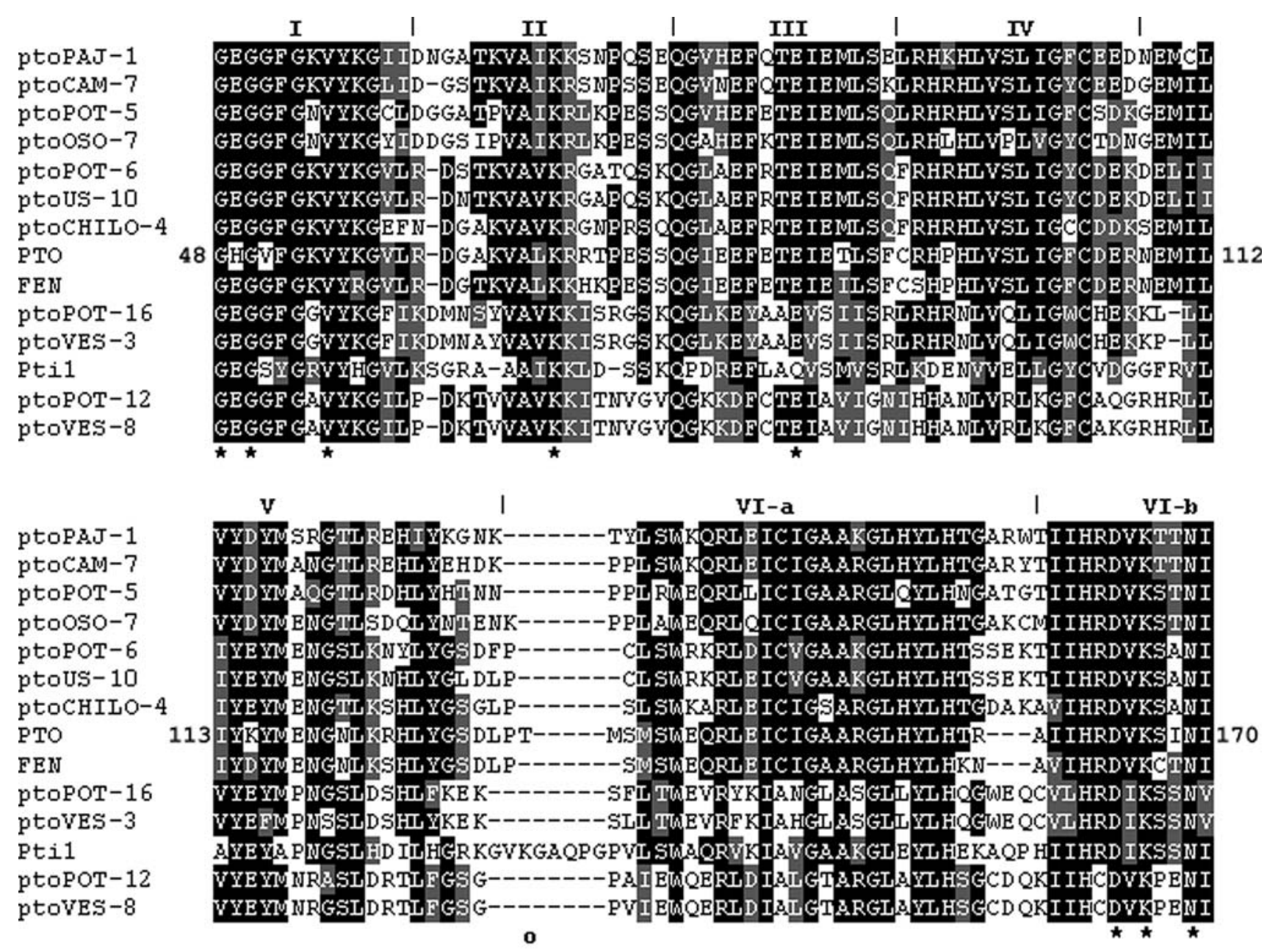

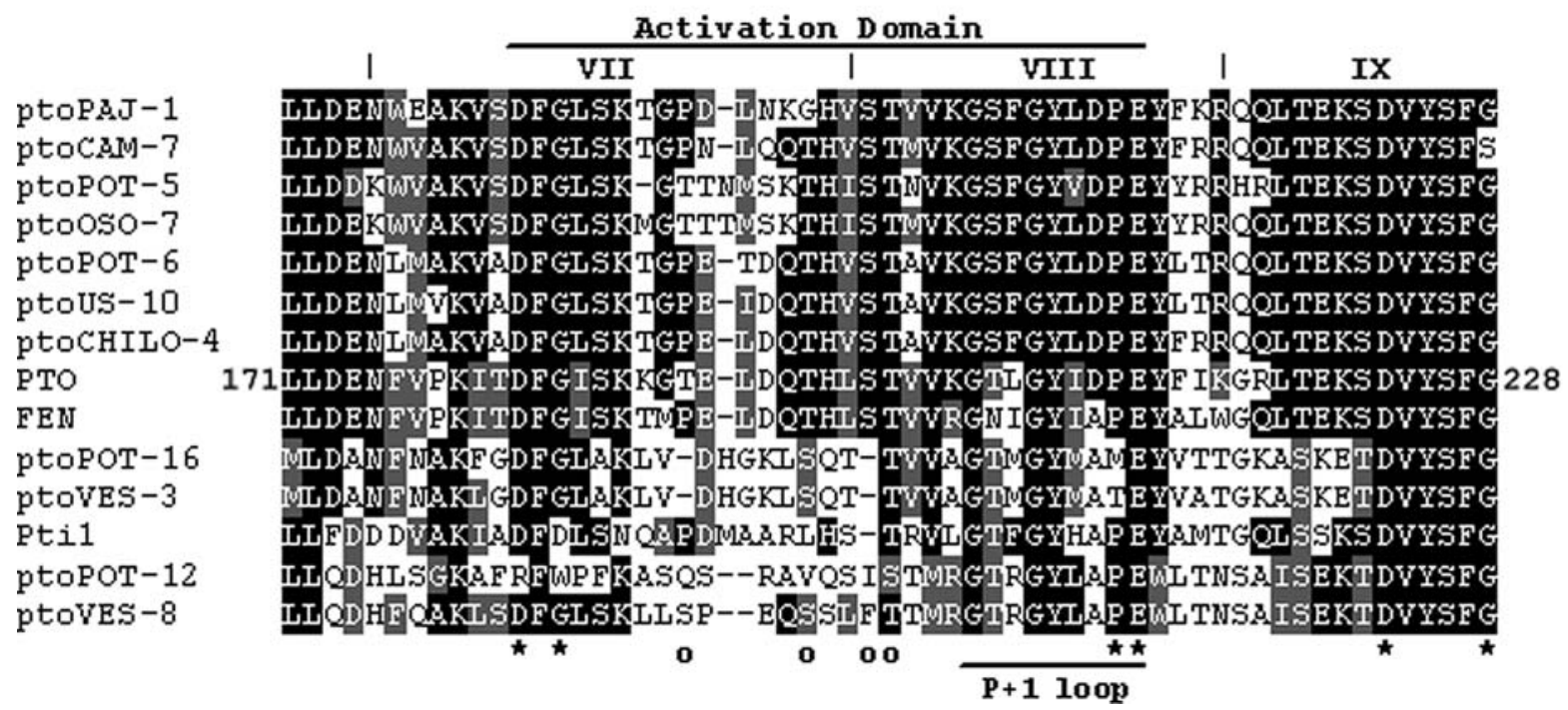

Fig. 2 Clustal X multiple-amino acid sequence alignment of strawberry STKs with the corresponding region of tomato R proteins Pto, Fen, and Pti1. Conserved subdomains (labeled with roman numerals), activation domain, and $\mathrm{P}+1$ loop are indicated. Kinase consensus sequences in Pto at positions 48, 50, 55, 69, 84, 164, 166, 169, 182, 184, 210, 211, 223, and 228 are indicated by asterisks. Pto autophosphorylation sites are indicated by circles 


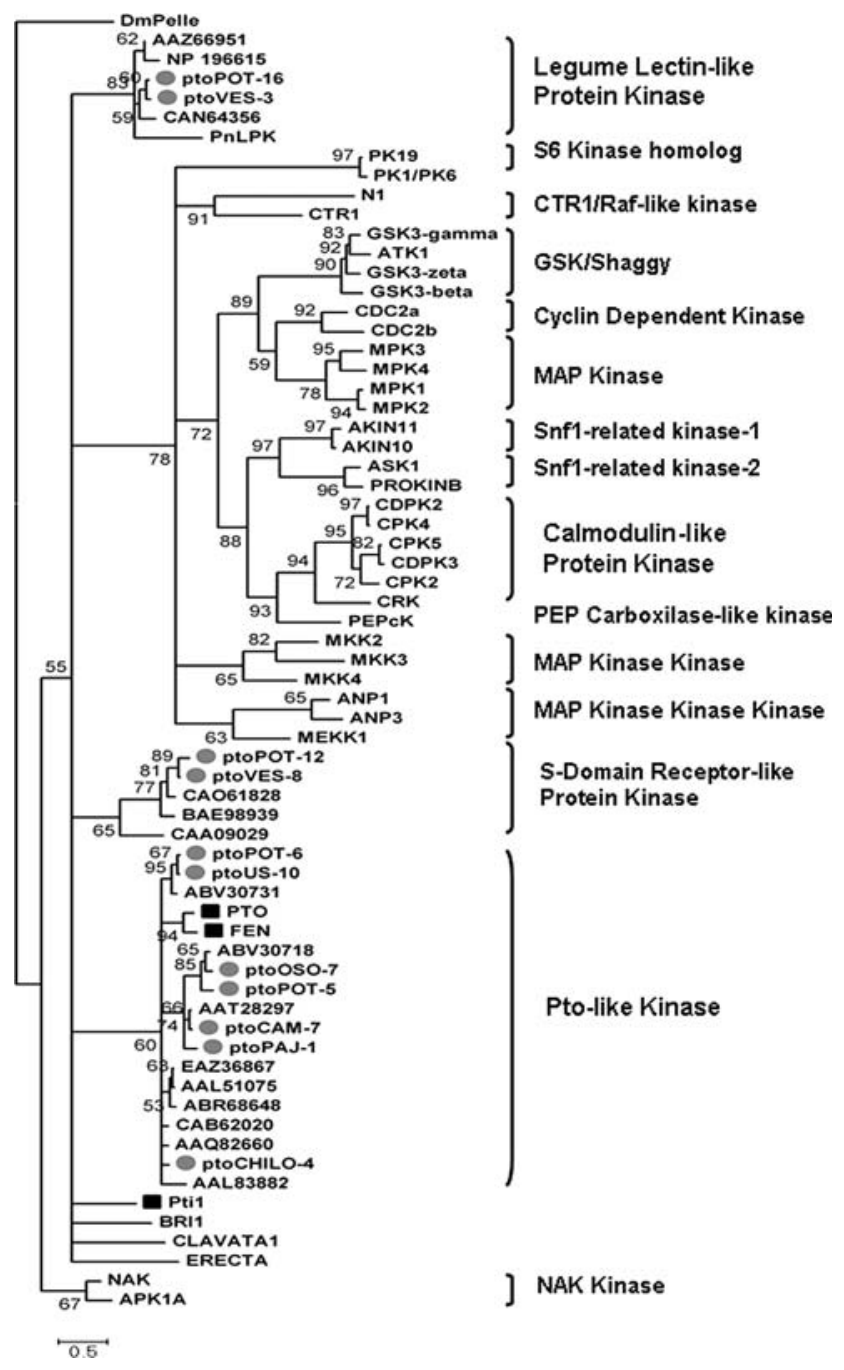

Fig. 3 Plant protein kinase tree inferred by ML analysis with the TREE-PUZZLE software. The tree was derived from comparison of the derived amino acid sequences of strawberry serine/threonine kinases (STKs; gray circles), 36 previously characterized STKs from A. thaliana (Hardie 1999), Pto, Fen, and Ptil R proteins from tomato (black squares), and putative STKs and Pto-like candidates from Arabidopsis (BAE98939, CAB62020, NP196615), Vitis (CAO61828, CAN64356), Prunus (ABV30718, ABV30731), Rosa (AAT28297), Brassica (AAZ66951), Capsicum (AAQ82660), Cucumis (AAL83882), Musa (ABR68648), Oryza (EAZ36867), Triticum (AAL51075), Zea (CAA09029), and Populus (PnLPK). As by Vallad et al. (2001), the Drosophila STK Pelle was used to root the tree. Values on tree branches represent the PUZZLE support values

abolished Pto-avrPto interaction in yeast hybrid system assays.

All strawberry Pto-like sequences were closely related to Pto and Fen, but the higher identity scores were found against other Pto-like candidates from both monocots and dicots (Table 1). The fact that strawberry Pto-like STKs were more closely related to Pto-like kinases of other species than to each other reveals that orthologues are more similar than paralogues, suggesting that these kinases share
Table 2 Comparison of strawberry Pto-like sequences with Pto and four of its paralogues

\begin{tabular}{|c|c|c|c|c|c|c|c|c|c|c|}
\hline & \multicolumn{2}{|c|}{$\mathrm{A}^{\mathrm{a}}$} & \multicolumn{2}{|c|}{$\mathrm{B}^{\mathrm{b}}$} & \multirow[t]{2}{*}{ Indel $^{\mathrm{c}} 159$} & \multicolumn{5}{|l|}{$E^{d}$} \\
\hline & 55 & 94 & 76 & 78 & & 202 & 204 & 205 & 207 & 209 \\
\hline PTO & V & $\mathrm{H}$ & $\mathrm{S}$ & G & & K & $\mathrm{T}$ & $\mathrm{L}$ & $\mathrm{Y}$ & D \\
\hline FEN & $\mathrm{V}$ & $\mathrm{H}$ & S & G & & $\mathrm{R}$ & $\mathrm{N}$ & I & $\mathrm{Y}$ & A \\
\hline LpimPth2 & D & $\mathrm{H}$ & $\mathrm{P}$ & $\mathrm{G}$ & & Q & S & I & $\mathrm{Y}$ & D \\
\hline LpimPth3 & $\mathrm{V}$ & $\mathrm{H}$ & Q & $\mathrm{S}$ & & $\mathrm{K}$ & $\mathrm{T}$ & $\mathrm{F}$ & $\mathrm{Y}$ & D \\
\hline LpimPth4 & $\mathrm{V}$ & $\mathrm{H}$ & $\mathrm{S}$ & $\mathrm{G}$ & & $\mathrm{K}$ & $\mathrm{T}$ & $\mathrm{L}$ & $\mathrm{Y}$ & D \\
\hline ptoPAJ-1 & V & $\mathrm{H}$ & $\mathrm{E}$ & G & ARW & $\mathrm{K}$ & $\mathrm{S}$ & $\mathrm{F}$ & $\mathrm{Y}$ & D \\
\hline ptoCAM-7 & $\mathrm{V}$ & $\mathrm{H}$ & $\mathrm{E}$ & G & ARY & $\mathrm{K}$ & S & $\mathrm{F}$ & $\mathrm{Y}$ & $\mathrm{D}$ \\
\hline ptoPOT-5 & V & $\mathrm{H}$ & $\mathrm{S}$ & $\mathrm{G}$ & ATG & $\mathrm{K}$ & $\mathrm{S}$ & $\mathrm{F}$ & Y & D \\
\hline ptoOSO-7 & V & $\mathrm{H}$ & $\mathrm{S}$ & G & AKC & $\mathrm{K}$ & $\mathrm{S}$ & $\mathrm{F}$ & $\mathrm{Y}$ & $\mathrm{D}$ \\
\hline ptoPOT-6 & V & $\mathrm{H}$ & $\mathrm{K}$ & G & SEK & $\mathrm{K}$ & $\mathrm{S}$ & $\mathrm{F}$ & $\mathrm{Y}$ & D \\
\hline ptoUS-10 & V & $\mathrm{H}$ & $\mathrm{K}$ & $\mathrm{G}$ & SEK & $\mathrm{K}$ & $\mathrm{S}$ & $\mathrm{F}$ & $\mathrm{Y}$ & D \\
\hline ptoCHILO-4 & V & $\mathrm{H}$ & Q & G & DAK & $\mathrm{K}$ & $\mathrm{S}$ & $\mathrm{F}$ & $\mathrm{Y}$ & D \\
\hline
\end{tabular}

${ }^{a}$ Residues V55 and H94, identified as important for resistance by ethyl methane sulfonate mutagenesis (Scofield et al. 1996)

b Residues S76 and G78, identified by the DNA-shuffling approach, provide specificity in binding AvrPtoB but do not affect binding to AvrPto (Bernal et al. 2005)

c Indel, insertion-deletion

${ }^{\mathrm{d}}$ Positions inside the $\mathrm{P}+1$ loop that are important for AvrPto binding specificity, identified by swaps between Pto and Fen together with site-directed mutagenesis (Scofield et al. 1996; Frederick et al. 1998) and by alanine substitution analysis (Rathjen et al. 1999)

a common evolutionary origin (Bogdanove 2002). This finding, together with the fact that Pto-like sequences clustered with both monocot and dicot Pto-like STKs, raises the possibility that the origin of this kind of PK occurred prior to the divergence between monocot and dicot plants.

The source of genetic variation within $R$ genes has been the subject of a great deal of speculation. Different genetic mechanisms have been proposed for the evolution of $R$ genes, such as recombination, unequal crossing-over, gene conversion, and point mutations (Michelmore and Meyers 1998). Pto analogues from wild and cultivated strawberry species showed considerable variation.

The ratio of nonsynonymous-to-synonymous substitutions $<1$ and the fact that no recombination was detected by GENCONV analysis indicated that the gradual accumulation of point mutations is the primary force generating variability. All these results suggest that the sequences may be subjected to purifying rather than diversifying selection, although we cannot rule out that positive selection could have been detected if we had included other segments of the protein sequences.

Additionally, neutrality tests failed to detect evidence of nonneutral evolution in these strawberry genes. Other indications that purifying selection may be operating on 
this type of $R$ gene are the natural variation of Pto and the structure/function relationships among Pto and other kinases. The catalytic core of kinases consists of 12 uninterrupted subdomains folded into a bilobal structure (Hanks and Hunter 1995), 9 of which are fully conserved in the cloned portion of strawberry STKs. Additionally, of the $\sim 300$ residues, 12 are essentially invariant among kinases, as observed among strawberry clones. Furthermore, functional studies of the Pto protein and its paralogues showed that almost $90 \%$ of the positions that knock out Avr recognition and/or signaling are invariant among alleles from natural populations (Sessa et al. 2000b; Wu et al. 2004; De Vries et al. 2006). Bernal et al. (2005) reported that the correlation between phenotypes and variation generated by DNA shuffling was similar to the natural variation observed between orthologues of Pto from Lycopersicon spp. Nevertheless, the $\mathrm{P}+1$ loop appears to be the region that experienced maintenance of polymorphism as long as the residues were hydrophobic or positively charged amino acids (Rose et al. 2007). In Pto, perturbing the activation segment by substituting the phosphorylated residues at positions 204, 205, and 207 with negatively charged amino acids, such as aspartic acid and glutamic acid, resulted in constitutively elicited cell death (Rathjen et al. 1999). We may speculate that this "polymorphism maintenance" could contribute to preventing Avr from escaping detection by Pto (Dangl and Jones, 2001).

Using an ethyl methane sulfonate mutagenesis approach, Scofield (1996) characterized residues V55 and H94 and showed that mutations at these positions disrupted the interaction Pto-AvrPto in yeast and abrogated Pto-mediated resistance. Table 2 reports that the latter residues are fully conserved in Pto-like strawbery clones.

Bernal et al. (2005) also identified, by DNA shuffling, residues that are involved in binding specificity to AvrPtoB but do not affect binding to AvrPto. Of these residues, G78 but not S76 is conserved in all strawberry sequences. This finding is consistent with previous results (Bernal et al. 2005) suggesting that residue S76 is subjected to divergent selection as determined by ML analysis of several Pto orthologues and paralogues. Results summarized in Table 2 also show that the strawberry Pto homologues present fewer polymorphisms at the indicated residues than Pto and its paralogues, suggesting that convergent processes prevail over divergent ones. Attempts to test whether the strawberry Pto-like genes support the hypotheses about how the guardian hypothesis operates were inconclusive, as the sequences assumed to be involved in the recognition of Avr protein present a rather high degree of conservation and, therefore, may not prevent Avr from escaping recognition.

Finally, Vleeshouwers et al. (2001) analyzed the phylogenetic relationships between 32 Pto-like sequences from six Solanum species together with tomato Pto, Fen, and other Pto-like sequences from many plant species. Based on the amino acid identity and distribution of insertions and deletions (indels), they distinguished nine STK classes. Class IX, containing all Pto homologues from non-solanum species, was distinguished by a three-amino acid insertion at position 159 of the Pto protein. We also observed a similar indel motif at the same position in strawberry sequences. This information suggests that the three-amino acid "indel" may have occurred prior to the divergence of solanaceous from other species. Thus, the Pto gene family as a whole is ancient and diverse.

In summary, homology-based PCR cloning of STK sequences proved to be succesful in strawberry and its related wild species. Also, this finding represents the first report of Pto-like candidates in strawberry. Taken together, these results provide the first insight into strawberry STKs, and their structure and evolution, and uncover a set of Ptolike candidates of potential use in genetic mapping and RNA interference functional essays.

Acknowledgments This paper was partially supported by Grants PICTO 04-759 BID 1728/OC-AR and CIUNT 26/D346. The work was carried out in compliance with the current laws regulating genetic experimentation in Argentina. G.M.Z. is a fellow of CONICET, and A.P.C. and J.C.D.R. are members of CONICET.

\section{References}

Aarts MG, te Lintel Hekkert B, Holub BT, Beynon JL, Stiekema WJ, Pereira A (1998) Identification of R-gene homologous DNA fragments genetically linked to disease resistance loci in Arabidopsis thaliana. Mol Plant-Microbe Interact 11:251-258

Altschul SF, Gish W, Miller W, Myers EW, Lipman DJ (1990) Basic local alignment search tool. J Mol Biol 215:403-410

Bernal A, Pollack J, Pan Q, Rose L, Kozik A (2005) Functional analysis of the plant resistance gene Pto using DNA shuffling. J Biol Chem 280:23073-23083

Bogdanove AJ (2002) Pto update: recent progress on an ancient plant defence response signalling pathway. Mol Plant Pathol 3:283288

Chang JH, Tai YS, Bernal AJ, Lavelle DT, Staskawicz BJ (2002) Functional analyses of the Pto resistance gene family in tomato and the identification of a minor resistance determinant in a susceptible haplotype. Mol Plant-Microbe Interact 15:281-291

Chen X, Shang J, Chen D, Lei C, Zou Y, Zhai W Liu G, Xu J, Ling Z, Ma B, Wang Y, Zhao X, Li S, Zhu L (2006) A B lectin receptor kinase gene conferring rice blast resistance. Plant J 46:794-804

Cui Y, Bi Y, Brugière N, Arnoldo M, Rothstein S (2000) The S locus glycoprotein and the $\mathrm{S}$ receptor kinase are sufficient for selfpollen rejection in Brassica. Proc Natl Acad Sci USA 97:37133717

Dangl JL, Jones JD (2001) Plant pathogens and integrated defence responses to infection. Nature 411:826-833

Dayhoff MO, Schwartz RM, Orcutt BC (1978) A model of evolutionary change in proteins. Matrices for detecting distant relationships. In: Dayhoff MO (ed) Atlas of protein sequence and structure. Vol. 5. National Biomedical Research Foundation, Washington, DC, pp 345-358 
Denoyes-Rothan B, Guérin G, Lerceteau-Köller E, Risser G (2005) Inheritance of resistance to Colletotrichum acutatum in Fragaria $\times$ ananassa. Phytopathology 95:405-412

DeVries JS, Andriotis VME, Wu AJ, Rathjen JP (2006) Tomato Pto encodes a functional $\mathrm{N}$-myristoylation motif that is required for signal transduction in Nicotiana benthamiana. Plant J 45:31-45

Ellis J, Dodds P, Pryor T (2000) Structure, function and evolution of plant disease resistance genes. Curr Opin Plant Biol 3:278-284

Flor HH (1956) The complementary genic systems in flax and flax rust. Adv Genet 8:29-54

Fluhr R (2001) Sentinels of disease. Plant resistance genes. Plant Physiol 127:1367-1374

Frederick RD, Thilmony RL, Sessa G, Martin GB (1998) Recognition specificity for the bacterial avirulence protein AvrPto is determined by Thr-204 in the activation loop of the tomato Pto kinase. Mol Cell 2:241-245

Fu YX, Li WH (1993) Statistical tests of neutrality of mutations. Genetics 133:693-709

Galletta GJ, Smith BJ, Gupton CL (1993) Strawberry parent clones US70, US159, US292, and US438 resistant to anthracnose crown rot. Hortscience 28:1055-1056

Hammond-Kosack KE, Jones JDG (1997) Plant disease resistance genes. Annu Rev Plant Physiol Plant Mol Biol 48:575-607

Hanks SK, Hunter T (1995) Protein kinases 6. The eukaryotic protein kinase superfamily: kinase (catalytic) domain structure and classification. FASEB J 9:576-596

Hanks SK, Quinn AM (1990) Protein kinase catalytic domain sequence database: identification of conserved features of primary structure and classification of family members. Methods Enzymol 200:38-62

Hardie DG (1999) Plant protein serine/threonine kinases: classification and functions. Annu Rev Plant Physiol Mol Biol 50:97-131

Haymes KM, Henken B, Davis TM, Van de Weg WE (1997) Identification of RAPD markers linked to a Phytophthora fragariae resistance gene (Rpf1) in the cultivated strawberry. Theoret Appl Genet 94:1097-1101

Hebsgaard SM, Korning PG, Tolstrup N, Engelbrecht J, Rouze P, Brunak S (1996) Splice site prediction in Arabidopsis thaliana DNA by combining local and global sequence information. Nucleic Acids Res 24:3439-3452

Hofmann K, Bucher P, Falquet L, Bairoch A (1999) The PROSITE database, its status in 1999. Nucleic Acids Res 27: 215-219

Joyeux A, Fortin MG, Mayerhofer R, Good AG (1999) Genetic mapping of plant disease resistance gene homologues using a minimal Brassica napus L. population. Genome 42:735-743

Kanazin V, Marek LF, Shoemaker RC (1996) Resistance gene analogs are conserved and clustered in soybean. Proc Natl Acad Sci USA 93:11746-11750

Kim YJ, Lin NC, Martin GB (2002) Two distinct Pseudomonas effector proteins interact with the Pto kinase and activate plant immunity. Cell 109:589-598

Korber B (2000) HIV signature and sequence variation analysis. In: Rodrigo AG, Learn GH (eds) Computational analysis of HIV molecular sequences. Kluwer Academic, Dordrecht, Netherlands, pp 55-72

Leister D, Ballvora A, Salamini F, Gebhardt C (1996) A PCR-based approach for isolating pathogen resistance genes from potato with potential for wide application in plants. Nat Genet 14:421442

Lerceteau-Kohler E, Roudeillac P, Markocic M, Guerin G, Praud K, Denoyes-Rothan B (2002) The use of molecular markers for durable resistance breeding in the cultivated strawberry (Fragaria $x$ ananassa). Acta Hort 567:615-618

Loh YT, Martin GB (1995) The Pto bacterial resistance gene and the Fen insecticide sensitivity gene encode functional protein kinases with serine/threonine specificity. Plant Physiol 108:1735-1739

Martin GB (1999) Functional Analysis of plant disease resistance genes and their downstream effectors. Curr Opin Plant Biol 2:273-279

Martin GB, Brommonschenkel SH, Chunwongse J, Frary A, Ganal MW, Spivey R, Wu T, Earle ED, Tanksley SD (1993) Mapbased cloning of a protein kinase gene conferring disease resistance in tomato. Science 262:1432-1435

Martin GB, Frary A, Wu T, Brommonschenkel S, Chunwongse J, Earle ED, Tanksley SD (1994) A member of the tomato Pto gene family confers sensitivity to fenthion resulting in rapid cell death. Plant Cell 6:1543-1552

Martin GB, Bogdanove AJ, Sessa G (2003) Understanding the function of plant disease resistance proteins. Annu Rev Plant Biol 54:23-61

Martínez-Zamora MG, Castagnaro AP, Diaz-Ricci JC (2004) Isolation and diversity analysis of resistance gene analogues (RGAs) from cultivated and wild strawberries. Mol Genet Genomics 272:480-487

McHale L, Tan X, Koehl P, Michelmore RW (2006) Plant NBS-LRR proteins: adaptable guards. Genome Biol 7:212

Michelmore RW, Meyers BC (1998) Clusters of resistance genes in plants evolve by divergent selection and a birth-and-death process. Genome Res 8:1113-1130

Morrisette PM (1989) The evolution of policy responses to stratospheric ozone depletion. Nat Resources J 29:793-820

Nei M, Gojobori T (1986) Simple methods for estimating the numbers of synonymous and nonsynonymous nucleotide substitutions. Mol Biol Evol 3:418-426

Nishiguchi M, Yoshida K, Sumizono T, Tazaki K (2002) A receptorlike protein kinase with a lectin-like domain from lombardy poplar: gene expression in response to wounding and characterization of phosphorylation activity. Mol Genet Genomics 267:506-514

Pan Q, Wendel J, Fluhr R (2000) Divergent evolution of plant NBSLRR resistance gene homologues in dicot and cereal genomes. J Mol Evol 50:203-213

Parker JE, Coleman MJ, Szabo V, Frost LN, Schmidt R, van der Biezen EA, Moores T, Dean C, Daniels MJ, Jones JDG (1997) The arabidopsis downy mildew resistance gene RPP5 shares similarity to the Toll and interleukin- 1 receptors with $N$ and $L 6$. Plant Cell 9:879-894

Pedley KF, Martin GB (2003) Molecular basis of Pto-mediated resistance to bacterial speck disease in tomato. Annu Rev Phytopathol 41:215-243

Peraza-Echeverría S, James-Kay A, Canto-Canché B, Castillo-Castro E (2007) Structural and phylogenetic analysis of Pto-type disease resistance gene candidates in banana. Mol Genet Genomics 278:443-453

Qin Y, Texeira da Silva J, Zhang L, Zhang Sh (2008) Transgenic strawberry: state of the art for improved traits. Biotechnol Adv 26:219-232

Rathjen JP, Chang JH, Staskawicz BJ, Michelmore RW (1999) Constitutively active Pto induces a Prf-dependent hypersensitive response in the absence of avrPto. EMBO J 18:3232-3240

Richter T, Ronald P (2002) The evolution of disease resistance genes. Plant Mol Biol 42:195-204

Rose LE, Michelmore RW, Langley CH (2007) Natural variation in the Pto pathogen resistance gene within species of wild tomato (Lycopersicon): II. Population genetics of Pto. Genetics 175:1307-1319

Rozas J, Sánchez-DelBarrio JC, Messegyer X, Rozas R (2003) DnaSP, DNA polymorphism analyses by the coalescent and other methods. Bioinformatics 19:2496-2497 
Ryals JA, Neuenschwander UH, Willits MG, Molina A, Steiner H-Y, Hunt MD (1996) Systemic acquired resistance. Plant Cell 8:1809-1819

Rudrabhatla P, Reddy MM, Rajasekharan R (2006) Genome-wide analysis and experimentation of plant serine/threonine/tyrosinespecific protein kinases. Plant Mol Biol 60:293-319

Sawyer S (1989) Statistical tests for detecting gene conversion. Mol Biol Evol 6:526-538

Schmidt HA, Strimmer K, Vingron M, von Haeseler A (2002) TREEPUZZLE: maximum likelihood phylogenetic analysis using quartets and parallel computing. Bioinformatics 18:502-504

Scofield SR, Tobias CM, Rathjen JP, Chang JH, Lavelle DT, Michelmore RW, Staskawicz BJ (1996) Molecular basis of genefor-gene specificity in bacterial speck disease of tomato. Science 274:2063-2065

Sessa G, D'Ascenzo M, Martin GB (2000a) The major site of the Pti1 kinase phosphorylated by the Pto kinase is located in the activation domain and is required for Pto-Pti1 physical interaction. Eur J Biochem 267:171-178

Sessa G, D’Ascenzo M, Martin GB (2000b) Thr38 and Ser198 are Pto autophosphorylation sites required for the AvrPto-Pto-mediated hypersensitive response. EMBO J 19:2257-2269

Shen KA, Meyers BC, Islam-Faridi N, Stelly DM, Michelmore RW (1998) Resistance gene candidates identified using PCR with degenerate oligonucleotide primers map to resistance gene clusters in lettuce. Mol Plant-Microbe Interact 11:815-823

Smith BJ, Black LL (1990) Morphological, cultural and pathogenic variation among Colletotrichum species isolated from strawberry. Plant Dis 74:69-76

Speulman E, Bouchez D, Holub EB, Beynon JL (1998) Disease resistance gene homologs correlate with disease resistance loci of Arabidopsis thaliana. Plant J 14:467-474
Tajima F (1989) Statistical method for testing the neutral mutation hypothesis by DNA polymorphism. Genetics 123:585-596

Tang X, Xie M, Kim YJ, Zhou J, Klessig DF, Martin GB (1999) Overexpression of Pto activates defense responses and confers broad resistance. Plant Cell 11:15-30

Thompson JD, Gibson TJ, Plewniak F, Jeanmougin F, Higgins DG (1997) The CLUSTAL_X windows interface: flexible strategies for multiple sequence alignment aided by quality analysis tools. Nucleic Acids Res 25:4876-4882

Vallad G, Rivkin M, Vallejos C, McClean P (2001) Cloning and homology modelling of a Pto-like protein kinase family of common bean (Phaseolus vulgaris L.). Theor Appl Genet 103:1046-1058

Vellicce GR, Díaz Ricci JC, Hernandez L, Castagnaro AP (2006) Enhanced resistance to Botrytis cinerea mediated by the transgenic expression of the chitinase gene ch5B in strawberry. Trans Res 15:57-68

Vleeshouwers VGAA, Martens A, van Dooijeweert W, Colon LT, Govers F, Kamoun S (2001) Ancient diversification of the Pto kinase family preceded speciation in Solanum. Mol Plant Microbe Interact 14:996-1005

Yu YG, Buss GR, Maroof MA (1996) Isolation of a superfamily of candidate disease-resistance genes in soybean based on a conserved nucleotide-binding site. Proc Natl Acad Sci USA 93:11751-11756

Wu A, Andriotis V, Durrant M, Rathjen JP (2004) A patch of surfaceexposed residues mediates negative regulation of immune signaling by tomato Pto kinase. Plant Cell 16:2809-2821

Zhou J, Tang X, Martin GB (1997) The Pto kinase conferring resistance to tomato bacterial speck disease interacts with proteins that bind a cis-element of pathogenesis-related genes. EMBO J 16:3207-3218 